\title{
Virtual force field algorithm for a behaviour-based autonomous robot in unknown environments
}

\begin{abstract}
The present paper describes a real-time motion-planning approach which lies in the integration of three techniques: fuzzy logic (FL), virtual force field (VFF), and boundary following (BF). The FL algorithm is used for velocity control based on sonar readings. The path-planning algorithm is based on the VFF and BF methods. The proposed navigation system differs from previous works in terms of using different algorithms for planning robot motion. Other improvements concern functional and computational aspects of the design and integration of the modules. The robot shows robust performance in complex situations and local minimum scenarios. Simulation results show the effectiveness of the developed system in various environments with long walls, U-shaped, maze-like, and other types of clutter.
\end{abstract}

Keyword: Boundary following; Fuzzy logic; Intelligent robots; Motion planning; Virtual force field 